\title{
Early vascular aging (EVA): consequences and prevention
}

\author{
Peter M Nilsson \\ Department of Clinical Sciences \\ Medicine, Lund University, University \\ Hospital, Malmö, Sweden
}

\begin{abstract}
It has often been recognized that a discrepancy exists during the clinical consultation between the chronological age of a patient on the one hand with the signs and symptoms of biological age that can be recorded on the other hand. In cardiovascular medicine this is obvious when a heavy smoker presents with features of early biological aging, for example skin appearance and impaired lung function. This could also be extrapolated to vascular function as the target for numerous cardiovascular risk factors, thereby increasing the risk of early cardiovascular disease (CVD). Both new and old treatment modalities can play a role for the prevention of early vascular aging, first of all smoking cessation and improved lifestyle in general, but later on also the use of drugs such as statins or agents that block the renin - angiotensin system (RAS). New classes of drugs are currently being tested for CVD prevention, including glitazones and rimonabant, even if adverse effects (heart failure and depression) might restrict their usefulness. Results from ongoing intervention studies will eventually cast new light on possibilities to prevent the development of vascular aging.
\end{abstract}

Keywords: aging, cardiovascular, diabetes, hypertension, risk factor, smoking, vascular

Cardiovascular risk in general is influenced by a number of risk factors and risk markers, some of them modifiable and possible to prevent, but other ones are nonmodifiable (Table 1). In subjects with a positive family history for cardiovascular disease (CVD) these risk factors or markers are often elevated as a result of genetic traits, eg, as mediated via familiar hypercholesterolemia, or socio-cultural factors, such as smoking habits, but not all of the risk is explained by conventional factors (Andersdottir et al 2002). There also seems to exist a special link between the risk of CVD in mothers and the corresponding risk in sons (Nilsson et al 2004; Sundquist and Li 2006), even adjusted for conventional risk factors in the offspring (Nilsson et al 2004). The causes for this association are largely unknown but could be complex. It is however well-known that a number of adverse psychosocial conditions and social stress can substantially influence the risk of CVD (Kaplan and Kiel 1993; Bairey et al 2002) and the clinical course of established disease and mortality, for example as recorded in people living in republics of the former Soviet Union (Plavinski et al 2003). One hypothesis to explain some aspects of early CVD in susceptible individuals is that of ongoing early cardio-vascular aging as part of a broader process of early biological aging (Nilsson 1996; Adams and White 2004). This concept could be helpful not only to explain the shifting incidence and prevalence rates of CVD in societies facing varying social and socio-economic difficulties, but also offer testable hypothesis in both animal and human experiments or clinical investigations. One example of this research would be to find novel long-term markers of the biological aging process, for example telomere length and corresponding telomerase activity (Aviv, 2003; Artandi 2006), even if the latter is normally highest in fetal life and early infancy - not in adulthood. 
Table I Major modifiable and nonmodifiable risk factors for cardiovascular disease

Modifiable risk factors

Smoking

Physical inactivity

Excessive alcohol consumption

Dietary factors

Dyslipidemia/Hyperlipidemia

Elevated LDL - cholesterol

Low HDL - cholesterol

Elevated triglycerides

Obesity (abdominal)

Diabetes mellitus/hyperglycemia

High blood pressure

Thrombogenic factors

Psychosocial stress

Poor sleep and relaxation

Adverse fetal growth pattern

Nonmodifiable risk factors

Age (chronological)

Gender

Ethnicity

Medical history of CVD or diabetes

Family history of CVD or diabetes

\section{What is vascular aging?}

Normal vascular aging is associated with a gradual change of the vascular structure and function, resulting in decreased arterial compliance and increased arterial stiffening (Edward et al 2002; Laurent et al 2006). This is influenced by agedependent structural and biochemical changes, eg, in the content of elastin and collagen in the vessel wall. Superimposed on this more or less normal process is pathological arterial vascular aging (atherosclerosis) with characteristic lesions and plaque formation (Hansson 2005), starting with subtle increased intima media thickening (IMT) in the vessel wall (Bots and Grobbee 2002). Another important aspect is the aging effects on impairment of endothelial-mediated vasodilatation and decrease in fibrinolysis.

In susceptible individuals the vascular aging process seems to take a more rapid course, eventually resulting in premature CVD manifestations, including coronary heart disease (CHD), myocardial infarction, stroke, and symptoms from peripheral arterial disease (PAD). Another aspect is the progressing vascular dementia with its clinical symptoms of impaired cognitive function, depression, and disturbed central regulation of physiological processes, eg, blood pressure regulation. This early (premature) vascular aging (EVA) is both influencing a more rapid course of normal age-dependent arterial changes and the superimposed pathological vascular aging based on atherosclerosis (Najjar et al 2005). The process is measurable directly by use of imaging devices such as ultrasound measurements of the arterial wall, for example in the carotid artery, the aorta, or the femoral artery, for evaluation of IMT and plaque formation. Another approach is to use physiological methods such as pulse wave velocity (PWV) or the arterial augmentation index (AI) based on pulse wave analysis, as markers of arterial stiffening. In addition the ankle-brachial pressure can be used as a marker of decreased peripheral arterial circulation. Other methods include the measurements of different biomarkers reflecting endothelial function (Jarvisalo et al 2006), as an important part of the vascular aging process, or the measurement of telomere length (Samani et al 2001), as outlined below. Some important features of EVA are listed in Table 2.

\section{Telomere attrition and vascular aging}

Telomeres are specialized chromatin structures that form the end cap of the DNA helix and are shortened by each cell division until the cell can divide no more. It has been repeatedly shown that telomere length, most often measured in peripheral blood cells is shorter (reflecting so called "mitotic senescence") in subjects with arterial aging, atherosclerosis, CHD, insulin resistance, impaired glucose tolerance and type 2 diabetes, or reporting poor lifestyle habits as reflected in obesity and excessive smoking (Obana et al 2003; Brouilette et al 2003; Ogami et al 2004; Benetso et al 2004; Gardner et al 2005; Valdes et al 2005; Cherkas et al 2006; Adaikalakoteswari et al 2007; Fitzpatrick et al 2007; Fuster et al 2007). Even psychosocial stress exposure has been shown to be associated with shorter telomere length (Epel et al 2004), as well as with increased risk of CVD (Kaplan and Keil 1993; Bairey et al 2002). Telomere length

Table 2 Features of early vascular ageing (EVA)

- Increased arterial stiffness and pulse wave velocity

- Impaired endothelial function and vasodilatation

- Chronic vascular inflammation

- Intima media thickness and early atherosclerosis

- Hemorheological disturbances of blood flow

- Capillary rarefaction and dysfunctional regulation

- Shorter telomere length/lower telomerase activity

- Impaired glucose and lipid metabolism

- Insulin resistance

- Oxidative stress

- Arterial calcification

- Increased deposition of matrix substances

- Small vessel degeneration in brain and kidney

- Increased left ventricular heart (LVH) load with hypertrophy 
is even a predictor of mortality risk in subjects aged 60 years or older (Cawthon et al 2003). No studies, however, have so far been able to show time-dependent changes in telomere length in relation to changes in vascular function or ultrasound imaging of arterial wall characteristics, for example the IMT, after repeated measurements. This could represent a fruitful approach in future studies of vascular changes in relation to age that is supposed to be heterogeneous in subjects at different CVD risk. Recently it was reported from the WOSCOP trial in middle-aged Scottish men that treatment with pravastatin reduced the risk of coronary heart disease associated with shorter telomeres (Brouilette et al 2007).

\section{Other molecular mechanisms associated with aging}

During mitochondrial metabolic activity, reactive oxidative species (ROS) are produced and increase the oxidative stress burden. This will negatively impact on aging processes, including the potential for DNA repair function. External causes for increased ROS will add to this, by the influence of smoking and certain aspects of food processing.

Current activities to characterize the genetic background for type 2 diabetes, based on so called whole genome scan approach (DGI 2007), will hopefully also shed light on the relationship with diabetes complications. Some of these complications, as influenced by hyperglycemia and other risk factors, could also be regarded as examples of early aging in poorly controlled diabetes.

In pre-hypertension and established hypertension the renin - angiotensin system (RAS) is often activated. It has been shown that induction of angiotensin II (Ang II), one important factor in the RAS activation, also promotes a cell growth arrest with phenotypic characteristics of cell senescence, such as enlarged cell shapes, increased senescence-associated beta-galactosidase positive staining cell, and depressed cell proliferation. Apoptotic changes are increased in senescent cells. The results from one experimental study suggested that cell apoptosis is possibly an important factor in the process of pathologic and physiologic senescence of endothelial cells as well as vascular aging (Shan et al 2007). This means that pharmacological agents blocking RAS and Ang II activity could theoretically slow down vascular aging processes.

\section{Investigations in a population-based cohort from SWEDEN}

One aspect of age-related changes in vascular structure is the development of IMT that can also be promoted by the influence of risk factors such as systolic blood pressure. Therefore,
IMT could be a surrogate marker for structural changes in the arterial vessel wall that resembles aging effects.

In the population-based Malmö Diet Cancer-Cardiovascular (MDC-CV) cohort study, from the city of Malmö in southern Sweden, we have access to ultrasound measurement of IMT, plaque prevalence rate, and estimation of carotid blood flow in over 6013 middle-aged men and women investigated at baseline in 1992-1995. Previous publications have reported on cross-sectional associations between the degree of IMT on the one hand with measures of insulin resistance (Hedblad et al 2000) or markers of inflammation and glucose metabolism, such as adiponectin (Nilsson et al 2006), on the other hand. Follow-up studies of the MDC-CV cohort have shown the predictive power of IMT for the incidence of stroke (Rosvall et al 2005a) and CHD (Rosvall et al 2005b). Increased IMT, reflecting early atherosclerosis, could also partly explain the link between low social class and low educational level with cardiovascular risk (Rosvall et al 2000, 2006). We currently do follow-up investigations after a mean duration of 14-years since the baseline investigation. Some of these subjects have, however, been treated pharmacologically for various risk factors and medical conditions in the meantime, a factor that has to be taken into account and adjusted for in all statistical analyses. One randomized controlled intervention study in a subgroup of MDC-CV showed the benefits of low-dose metoprolol for slowing of the IMT progression; the BCAPS study (Hedblad 2001). In another randomized, controlled intervention trial the effect of the insulin sensitizing agent rosiglitazone was tested for slowing of IMT progression in subjects with type- 2 diabetes or the insulin resistance syndrome (Hedblad et al 2007). In summary, the MDC-CV cohort offers interesting possibilities to further elucidate on the phenomenon of early vascular aging in relation to biological, lifestyle-related, and psychosocial risk factors. Investigations of genetic markers, telomere length, and arterial stiffness (pulse wave velocity, AI) are other research options currently being explored in this cohort, similar to the ASKLEPIOS Study in Belgium (Rietzschel et al 2007).

\section{The role of diabetes in early aging}

The incidence of type-2 diabetes typically increases with age, due to age-related changes in beta-cell function and insulin sensitivity, in conjunction with lowered physical activity and increase in body weight and relative fat mass. Increasing levels of hyperglycemia are associated with early CVD events (Danaei et al 2006), and this could be due either to the impact of hyperglycemia per se or its determinants (eg, insulin resistance) or consequences 
(advanced glycosylation end products; AGE). The risk could, however, also be a consequence of the impact of various CVD risk factors associated with hyperglycemia (insulin resistance), eg, dyslipidemia, elevated blood pressure, defects in fibrinolysis, chronic low-grade inflammation, etc. (Stamler et al 1993). It is therefore not unexpected that subjects with type-2 diabetes mellitus also experience an elevated CVD risk, relatively more pronounced in women than in men with diabetes $(\mathrm{Hu}$ et al 2005). For these well proven reasons established diabetes can be regarded as a model of early biological aging with accompanying early vascular aging that can be seen as both macro- and microvascular manifestations. In a few cases, even longstanding diabetes can however be encountered without severe complications, as described in the so-called "Golden Years Project" in the UK (Bain et al 2003). This could be a manifestation of either protective genes for example regulation of HDL cholesterol metabolism via enzymes such as CETP, healthy lifestyle, or effects of medical care and compliance with anti-diabetes treatment, eg, insulin therapy.

The role of AGE for arterial stiffening is important, as this is a mechanisms related to the glycosylation in many various proteins in different organs, thus explaining stiffening of not only vascular tissue but also for example lung tissue in relation to the effects of hyperglycemia.

\section{How to prevent early vascular aging?}

In subjects with increased risk and a positive family history of early manifestations of CVD it is advisable to screen for and treat all conventional risk factors such as smoking, hypertension, hyperlipidemia and impaired glucose tolerance or type 2 diabetes, according to current guidelines (Graham et al 2007). Smoking is a deleterious risk factor, not only for coronary heart disease in a dose-response fashion according to the INTERHEART study (Teo et al 2006), but also for vascular dysfunction in general and the risk of increased insulin resistance and type 2 diabetes (Rimm et al 1995). New pharmacological treatment alternatives, for example use of the partial nicotine receptor-agonist varenicline (Jorenby et al 2006), might help smokers to quit their deleterious nicotine dependence. Lifestyle improvements in general (physical exercise and a balanced diet) are also useful components for cardiovascular protection, as shown for fruit and vegetables and modest alcohol intake in the INTERHEART study. This should be applied to many people with conventional risk factors below cut-off levels in current guidelines, eg, for blood pressure, cholesterol or glucose, of increased arterial stiffness is present, being one marker of vascular aging.
Other evidence-based treatment modalities include the use of ACE-inhibitors for prevention of CVD manifestations based on atherosclerosis in risk patients (Dagenais et al 2006), as well as statin therapy (CTT 2005), according to recent large meta-analyses. One interesting possibility is that treatment with pravastatin seems to reduce the risk for coronary heart disease events in middle-aged men with short telomeres, as described in the WOSCOP trial (Brouilette et al 2007). It is still largely unproven if the angiotensin-2 (AT1) receptor blockers (ARB) can offer the same, more, or less vascular protection as compared to the more studied class of ACEinhibitors (Dagenais et al 2006). However, in the large-scale ONTARGET trial, more than 25,000 subjects with hypertension and associated risk factors have been randomized to treatment either by the ACE-inhibitor ramipril or the ARB telmisartan, or to the combination of the two drugs, for prevention of fatal and nonfatal CVD endpoints (Teo et al 2004). This trial is of substantial clinical importance and expected to be presented in 2008 .

The treatment of diabetes and its hyperglycemia should be based on a new treatment algorithm presented in 2006 by ADA and EASD (Nathan et al 2006), including a first step of lifestyle modification in combination with metformin. As an optional second step the use of insulin, sulphonylurea or glitazones can be advocated by appropriate need. How to work for a strict CVD risk factor control in all patients with diabetes is outlined in another example of new joint guidelines from the ESC/EASD in Europe (Rydén et al 2007). If all these preventive measures could be effectively applied, the risk of early (cardio-) vascular aging could be counteracted and the progression slowed in these high-risk patients.

\section{Conclusions}

The concept of early (premature) vascular aging (EVA) represents a potentially fruitful model for future research into pathophysiological mechanisms behind early manifestations of CVD. For example, more research is needed to elucidate on the gene-environment interaction behind vascular and endothelial dysfunction, eg, the interaction between the genetic determinant of nitric oxide production in the endothelial wall by nitric oxide synthetase (eNOS) in relation to smoking exposure (Leeson et al 2002). We also need a more thorough evaluation of different markers of early vascular aging for CVD risk prediction: Such markers could include different measures of arterial stiffness, a hallmark of early vascular aging in combination with endothelial dysfunction as evident in some patients with hypertension (Taddei et al 1997). New biomolecular markers such as telomere length 
(Aviv 2002) have to be evaluated in various intervention studies, both with lifestyle advice and with specific drug therapy. Such potentially beneficial drugs not only include well-established drugs for vascular protection like statins and ACE-inhibitors, but also newer drugs such as ARB, the endocannaboid receptor (CB1) antagonist rimonabant, and the PPAR-activating glitazones, even if some adverse effects (heart failure) could caution against a wide use of the latter class of drugs. Examples of randomized controlled trials exploring cardiovascular protection of these newer compounds include ONTARGET for ARB (Teo et al 2004), CRESCENDO for rimonabant (CRESCENDO 2005), and RECORD for rosiglitazon (Home et al 2005). During 2007 the revision of guidelines for cardiovascular prevention was published (Graham et al 2007). It is still an open question if the concept of early vascular aging will be directly addressed or not in future guidelines, but the risk factors contributing to this condition and their evidence-based treatment will certainly be. A larger question to solve is to understand and prevent many of the adverse psychosocial risk factors, and their lifestyle correlates that might speed up the process of early biological aging in general (Nilsson 2003; Chandola et al 2006). This is a question of efforts in public health policy making (Marmot 2006), and not a matter of drug treatment.

Finally, all physicians should educate their patients to healthy lifestyle to prevent or delay vascular aging, cardiovascular diseases, and to maintain a good quality of life and thereby increase their life expectancy.

\section{Acknowledgments}

I wish to thank Frej Fyhrqvist, Helsinki, Finland, Björn Dahlöf, Göteborg, Sweden, and Stéphane Laurent, Paris, France, for fruitful discussion on the EVA concept.

\section{References}

Adaikalakoteswari A, Balasubramanyam M, Ravikumar R, et al. 2007. Association of telomere shortening with impaired glucose tolerance and diabetic macroangiopathy. Atherosclerosis, 195:83-9.

Adams JM, White M. 2004. Biological ageing. A fundamental, biological link between socio-economic status and health? Eur J Pub Health, 14:331-4.

Andresdottir MB, Sigurdsson G, Sigvaldason H, et al. 2002. Reykjavik Cohort Study. Fifteen percent of myocardial infarctions and coronary revascularizations explained by family history unrelated to conventional risk factors. The Reykjavik Cohort Study. Eur Heart J, 23:1655-63.

Artandi SE. 2006. Telomeres, telomerase, and human disease. $N$ Engl J Med, 355:1195-7.

Aviv A. 2002. Telomeres, sex, reactive oxygen species, and human cardiovascular aging. J Mol Med, 80:689-95.

Aviv A, Levy D, Mangel M. 2003. Growth, telomere dynamics and successful and unsuccessful human aging. Mech Ageing Dev, 124:829-37.

Bain SC, Gill GV, Dyer PH, et al. 2003. Characteristics of type 1 diabetes of over 50 years duration (the Golden Years Cohort). Diabet Med, 20:808-11.
Bairey Merz CN, Dwyer J, Nordstrom CK, et al. 2002. Psychosocial stress and cardiovascular disease: pathophysiological links. Behav Med, 27:141-7.

Benetos A, Gardner JP, Zureik M, et al. 2004. Short telomeres are associated with increased carotid atherosclerosis in hypertensive subjects. Hypertension, 43:182-5.

Bots ML, Grobbee DE. 2002. Intima media thickness as a surrogate marker for generalised atherosclerosis. Cardiovasc Drugs Ther, 16:341-51.

Brouilette S, Singh RK, Thompson JR, et al. 2003. White cell telomere length and risk of premature myocardial infarction. Arterioscler Thromb Vasc Biol, 23:842-6.

Brouilette SW, Moore JS, McMahon AD, et al. 2007. West of Scotland Coronary Prevention Study Group. Telomere length, risk of coronary heart disease, and statin treatment in the West of Scotland Primary Prevention Study: a nested case-control study. Lancet, 369:107-14.

Cawthon RM, Smith KR, O'Brien E, et al. 2003. Association between telomere length in blood and mortality in people aged 60 years or older. Lancet, 361:393-5.

Chandola T, Brunner E, Marmot M. 2006. Chronic stress at work and the metabolic syndrome: prospective study. BMJ, 332:521-5.

Cherkas LF, Aviv A, Valdes AM, et al. 2006. The effects of social status on biological aging as measured by white-blood-cell telomere length. Aging Cell, 5:61-5.

[CTT] Cholesterol Treatment Trialists' Collaborators. 2005. Efficacy and safety of cholesterol-lowering treatment: prospective meta-analysis of data from 90,056 participants in 14 randomized trials of statins. Lancet, 366:1267-78.

[CRESCENDO] Comprehensive Rimonabant Evaluation Study of Cardiovascular ENDpoints and Outcomes; Sanofi-Aventis. 2005. Comprehensive Rimonabant Evaluation Study of Cardiovascular ENDpoints and Outcomes (CRESCENDO) [online]. Accessed on October 10, 2007. URL: http://clinicaltrials.gov/ct/show/ NCT00263042? order $=1$.

Dagenais GR, Pogue J, Fox K, et al. 2006. Angiotensin-converting-enzyme inhibitors in stable vascular disease without left ventricular systolic dysfunction or heart failure: a combined analysis of three trials. Lancet, 368:581-8.

Danaei G, Lawes CM, Vander Hoorn S, et al. 2006. Global and regional mortality from ischaemic heart disease and stroke attributable to higher-than-optimum blood glucose concentration: comparative risk assessment. Lancet, 368:1651-9.

[DGI] Diabetes Genetics Initiative of Broad Institute of Harvard and IMT, Lund University and NOVARTIS Institutes for Biomedical Research. 2007. Genome-wide association analysis identifies three novel loci for type-2 diabetes and one for triglyceride levels. Science, 316:1331-6.

Edward G. Lakatta EG, Najjar SS. 2002. Vascular aging: An emerging new global cardiovascular risk. The Valencia Forum [abstract online]. Accessed on October 10, 2007. URL: http://www.valenciaforum. com/Keynotes/el.html.

Epel ES, Blackburn EH, Lin J, et al. 2004. Accelerated telomere shortening in response to life stress. Proc Natl Acad Sci USA, 101:17312-15.

Fitzpatrick AL, Kronmal RA, Gardner JP, et al. 2007. Leukocyte telomere length and cardiovascular disease in the Cardiovascular Health Study. Am J Epidemiol, 165:14-21.

Fuster JJ, Diez J, Andrès V. 2007. Telomere dysfunction and hypertension. J Hypertens, 25:2185-92.

Gardner JP, Li S, Srinivasan SR, et al. 2005. Rise in insulin resistance is associated with escalated telomere attrition. Circulation, 111:2171-7.

Graham I, Atar D, Borch-Johnsen K, et al. 2007. European guidelines on cardiovascular disease prevention in clinical practice: executive summary: Fourth Joint Task Force of the European Society of Cardiology and Other Societies on Cardiovascular Disease Prevention in Clinical Practice (Constituted by representatives of nine societies and by invited experts). Eur Heart J, 28:2375-414.

Hansson GK. 2005. Inflammation, atherosclerosis, and coronary artery disease. N Engl J Med, 352:1685-95. 
Hedblad B, Nilsson P, Eriksson K-F, et al. 2000. Risk factor pattern and prevalence of atherosclerotic disease in subjects with the metabolic syndrome. Data from the Malmö Diet and Disease Study. Diabetic Medicine, 17:299-307.

Hedblad B, Wikstrand J, Janzon L, et al. 2001. Low-dose metoprolol CR/XL and fluvastatin slow progression of carotid intima-media thickness: Main results from the Beta-Blocker Cholesterol-Lowering Asymptomatic Plaque Study (BCAPS). Circulation, 103:1721-6.

Hedblad B, Nilsson PM, Zambanini A, et al. 2007. Rosiglitazone reduces carotid intima-media thickness progression over 1 year in patients with type 2 diabetes but not in non-diabetic people who have insulin resistance alone. J Internal Med, 261:293-305.

Home PD, Pocock SJ, Beck-Nielsen H, et al. 2005. Rosiglitazone Evaluated for Cardiac Outcomes and Regulation of Glycaemia in Diabetes (RECORD): study design and protocol. Diabetologia, 48:1726-35.

Hu G, Jousilahti P, Qiao Q, et al. 2005. Sex differences in cardiovascular and total mortality among diabetic and non-diabetic individuals with or without history of myocardial infarction. Diabetologia, 48:856-61.

Jarvisalo MJ, Juonala M, Raitakari OT. 2006. Assessment of inflammatory markers and endothelial function. Curr Opin Clin Nutr Metab Care, 9:547-52.

Jorenby DE, Hays JT, Rigotti NA, et al. 2006. Varenicline Phase 3 Study Group. Efficacy of varenicline, an alpha4beta2 nicotinic acetylcholine receptor partial agonist, vs placebo or sustained-release bupropion for smoking cessation: a randomized controlled trial. JAMA, 296:56-63.

Kaplan GA, Keil JE. 1993. Socio-economic factors and cardiovascular disease: a review of the literature. Circulation, 88:1973-98.

Laurent S, Cockkroft J, Van Bortel L, et al. 2006. Expert consensus document on arterial stiffness: methodological issues and clinical applications. Eur Heart J, 27:2588-605.

Leeson CP, Hingorani AD, Mullen MJ, et al. 2002. Glu298Asp endothelial nitric oxide synthase gene polymorphism interacts with environmental and dietary factors to influence endothelial function. Circ Res, 90:1153-8.

Marmot M. 2006. Health in an unequal world. Lancet, 368:2081-94.

Najjar SS, Scuteri A, Lakatta EG. 2005. Arterial aging: is it an immutable cardiovascular risk factor? Hypertension, 46:454-62.

Nathan DM, Buse JB, Davidson MB, et al. 2006. Management of hyperglycemia in type 2 diabetes: A consensus algorithm for the initiation and adjustment of therapy: a consensus statement from the American Diabetes Association and the European Association for the Study of Diabetes. Diabetes Care, 29:1963-72.

Nilsson P. 1996. Premature ageing - the link between psychosocial risk factors and disease? Med Hypotheses, 47:39-42.

Nilsson PM, Nilsson J-Å, Berglund G. 2004. Family burden of cardiovascular mortality - risk implications for offspring in a national register linkage study based upon the Malmö Preventive Project. J Intern Med, 255:229-35.

Nilsson PM, Engberg M, Nilsson J-Å, et al. 2005. Adverse social factors predict early ageing in middle-aged men and women: the Ebeltoft Health Study, Denmark. Scand J Public Health, 31:1-6.

Nilsson PM, Engström G, Hedblad B, et al. 2006. Plasma adiponectin levels are inversely associated with carotid intima media thickness and markers of insulin resistance in middle-aged men. ATVB, 26:2758-62.

Obana N, Takagi S, Kinouchi Y, et al. 2003. Telomere shortening of peripheral blood mononuclear cells in coronary disease patients with metabolic disorders. Intern Med, 42:150-3.

Ogami M, Ikura Y, Ohsawa M, et al. 2004. Telomere shortening in human coronary artery diseases. Arterioscler Thromb Vasc Biol, 24:546-50.
Plavinski SL, Plavinskaya SI, Klimov AN. 2003. Social factors and increase in mortality in Russia in the 1990s: prospective cohort study. BMJ, 326:1240-2

Rietzschel ER, De Buyzere ML, Bekaert S, et al. 2007. Asklepios Investigators. Rationale, design, methods and baseline characteristics of the Asklepios Study. Eur J Cardiovasc Prev Rehab, 14:179-91.

Rimm EB, Chan J, Stampfer MJ, et al. 1995. Prospective study of cigarette smoking, alcohol use, and the risk of diabetes in men. $B M J$, 310:555-9.

Rosvall M, Östergren PO, Hedblad B, et al. 2000. Occupational status, educational level, and the prevalence of carotid atherosclerosis in a general population sample of middle-aged Swedish men and women: results from the Malmo Diet and Cancer Study. Am J Epidemiol, 152:334-46.

Rosvall M, Janzon L, Berglund G, et al. 2005a. Incidence of stroke is related to carotid IMT even in the absence of plaque. Atherosclerosis, 179:325-31.

Rosvall M, Janzon L, Berglund G, et al. 2005b. Incident coronary events and case fatality in relation to common carotid intima-media thickness. J Intern Med, 257:430-7.

Rosvall M, Östergren PO, Hedblad B, et al. 2006. Socioeconomic differences in the progression of carotid atherosclerosis in middle-aged men and women with subclinical atherosclerosis in Sweden. Soc Sci Med, 62:1785-98.

Rydén L, Standl E, Bartnik M, et al. Task Force on Diabetes and Cardiovascular Diseases of the European Society of Cardiology (ESC); European Association for the Study of Diabetes (EASD). 2007. Guidelines on diabetes, pre-diabetes, and cardiovascular diseases: executive summary. The Task Force on Diabetes and Cardiovascular Diseases of the European Society of Cardiology (ESC) and of the European Association for the Study of Diabetes (EASD). Eur Heart J, 28:88-136.

Samani NJ, Boultby R, Butler R, et al. 2001. Telomere shortening in atherosclerosis. Lancet, 358:472-3.

Shan HY, Bai XJ, Chen XM. 2007. Apoptosis is involved in the senescence of endothelial cells induced by angiotensin II. Cell Biol Int, Sep 19; [Epub ahead of print]

Stamler J, Vaccaro O, Neaton JD, et al. 1993. Diabetes, other risk factors, and 12-yr cardiovascular mortality for men screened in the Multiple Risk Factor Intervention Trial. Diabetes Care, 16:434-44.

Sundquist K, Li X. 2006. Differences in maternal and paternal transmission of coronary heart disease. Am J Prev Med, 30:480-6.

Taddei S, Virdis A, Mattei P, et al. 1997 Hypertension causes premature aging of endothelial function in humans. Hypertension, 29:736-43.

Teo K, Yusuf S, Sleight P, et al. ONTARGET/TRANSCEND Investigators. 2004. Rationale, design, and baseline characteristics of 2 large, simple, randomized trials evaluating telmisartan, ramipril, and their combination in high-risk patients: the Ongoing Telmisartan Alone and in Combination with Ramipril Global Endpoint Trial/Telmisartan Randomized Assessment Study in ACE Intolerant Subjects with Cardiovascular Disease (ONTARGET/TRANSCEND) trials. Am Heart $J, 148: 52-61$.

Teo KK, Ounpuu S, Hawken S, et al. INTERHEART Study Investigators. 2006. Tobacco use and risk of myocardial infarction in 52 countries in the INTERHEART study: a case-control study. Lancet, 368:647-58.

Valdes AM, Andrew T, Gardner JP, et al. 2005. Obesity, cigarette smoking, and telomere length in women. Lancet, 366:662-4. 